\title{
Erlang Capacity of a Power Controlled CDMA System
}

\author{
Audrey M. Viterbi and Andrew J. Viterbi
}

\begin{abstract}
This paper presents an approach to the evaluation of the reverse link capacity of a CDMA cellular voice system which employs power control and a variable rate vocoder based on voice activity. It is shown that the Erlang capacity of CDMA is many times that of conventional analog systems and several times that of other digital multiple access systems.
\end{abstract}

\section{INTRODUCTION}

$\mathbf{F}$ OR any multiuser communication system, the measure of its economic usefulness is not the maximum number of users which can be serviced at one time, but rather the peak load that can be supported with a given quality and with availability of service as measured by the blocking probability (the probability that a new user will find all channels busy and hence be denied service, generally accompanied by a busy signal). Adequate service is usually associated with a blocking probability of 2 percent or less. The average traffic load in terms of average number of users requesting service resulting in this blocking probability is called the Erlang capacity of the system.

In virtually all existing multiuser circuit-switched systems, blocking occurs when all frequency slots or time slots have been assigned to a voice conversation or message. In code division multiple access (CDMA) systems, in contrast, users all share a common spectral frequency allocation over the time that they are active. Hence, new users can be accepted as long as there are receiver-processors to service them, independent of time and frequency allocations. We shall assume that a sufficient number of such processors is provided in the common base station such that the probability of a new arrival finding them all busy is negligible. Rather, blocking in CDMA systems will be defined to occur when the interference level, due primarily to other user activity, reaches a predetermined level above the background noise level of mainly thermal origin. While this interference-to-noise ratio could, in principle, be made arbitrarily large, when the ratio exceeds a given level (about $10 \mathrm{~dB}$ nominally), the interference increase per additional user grows very rapidly, yielding diminishing returns and potentially leading to instability. Consequently, we shall establish blocking in CDMA as the event that the total interference-to-background noise level exceeds $1 / \eta$ (where $\eta=0.1$ corresponding to $10 \mathrm{~dB}$ ), and we determine the Erlang capacity which results in a 1 percent blocking probability. We

Manuscript received March 16, 1992; revised November 16, 1992. Part of this paper was presented at the IEEE International Symposium on Information Theory, San Antonio, TX, January 17-22, 1993.

The authors are with Qualcomm Inc., San Diego, CA 92121-1617.

IEEE Log Number 9208658. emphasize, however, that this is a "soft blocking" condition, which can be relaxed as will be shown, as contrasted to the "hard blocking" condition wherein channels are all occupied.

Also, in conventional systems, a fraction of the time or frequency slots must be set aside for users to transmit requests for initiating service, and a protocol must be established for multiple requests when two or more users collide in simultaneously requesting service. In CDMA systems, even the users seeking to initiate access can share the common medium. Of course, they add to the total interference and hence lower the Erlang capacity to some degree. We shall demonstrate that this reduction is very small for initial access requests whose signaling time is on the order of a few percent of the average duration of a call or message.

Conclusions will be drawn regarding the relative increase in Erlang capacity of a direct sequence spread-spectrum CDMA system over existing FDMA and TDMA systems.

\section{CONVENTIONAL BLOCKING}

In conventional multiple access systems, such as FDMA and TDMA, traffic channels are allocated to users as long as there are channels available, after which all incoming traffic is blocked until a channel becomes free at the end of a call. The blocking probability is obtained from the classical Erlang analysis of the $M / M / S / S$ queue, where the first $M$ refers to a Poisson arrival rate of $\lambda$ calls/s; the second $M$ refers to exponential service time with mean $1 / \mu \mathrm{s} /$ call; the first $S$ refers to the number of servers (channels); and the second $S$ refers to the maximum number of users supported before blockage occurs.

The Erlang-B formula [1] gives the blocking probability under these conditions

$$
P_{\text {blocking }}=\frac{(\lambda / \mu)^{S} / S !}{\sum_{k=0}^{S}(\lambda / \mu)^{k} / k !}
$$

where $\lambda / \mu$ is the offered average traffic measured in Erlangs. Of course, the average active number of users equals $(\lambda / \mu)\left(1-P_{\text {blocking }}\right)$.

Thus, for the conventional AMPS system with $30 \mathrm{kHz}$ channels $K=7$ frequency reuse factor ${ }^{1}$ and 3 sectors, the number

\footnotetext{
${ }^{1}$ It should be noted that the reuse factor of 7 applies strictly only to North American analog systems. Digital TDMA systems have been proposed for other regions with lower reuse factors (4 or even 3 ). Field experience is not yet available, however, to support general use of such lowered reuse factors.
} 
of channels (servers) in $12.5 \mathrm{MHz}$ is

$$
S_{\text {AMPS }}=\frac{12.5 \mathrm{MHz}}{(30 \mathrm{kHz})(7)(3)}=19 \text { channel } / \text { sector } \text {. }
$$

Similarly, for a 3-slot TDMA system, which otherwise uses the same channelization, sectorization, and reuse factor as AMPS, and is generally called D-AMPS,

$$
S_{D-\mathrm{AMPS}}=57 \text { channel } / \text { sector } .
$$

The blocking probabilities $P$ (blocking) as a function of the average number of calls offered at any instant $\lambda / \mu$ are obtained from the Erlang-B formula. This establishes that the offered traffic Erlang capacity per sector for a blocking probability equal to 2 percent is respectively,

$$
\begin{aligned}
(\lambda / \mu)_{\mathrm{AMPS}} & =12.34 \text { Erlangs }, \\
(\lambda / \mu)_{D \text {-AMPS }} & =46.8 \text { Erlangs } .
\end{aligned}
$$

\section{CDMA REVERSE LINK ERLANG CAPACITY BOUNDS AND APPROXIMATIONS}

For the CDMA reverse link (or uplink), which is the limiting direction, blocking is defined to occur when the total collection of users both within the given sector (cell) and in other cells introduce an amount of interference density $I_{0}$ so great that it exceeds the background noise level $N_{0}$ by an amount $1 / \eta$, taken to be $10 \mathrm{~dB}$.

If there were always

1) a constant number of users $N_{u}$ in every sector,

2) each (perfectly power controlled) user were transmitting continually, and

3) required the same $E_{b} / I_{0}$ (under all propagation conditions),

then, as established in [2] and [3], the number of users $N_{u}$ would be determined by equating

$$
\begin{gathered}
\left.N_{u} \text { (Signal Power } / \text { User }\right)+ \text { Other Cell Interference } \\
+ \text { Thermal Noise }
\end{gathered}
$$

$=$ Total Interference

Taking

$W=$ spread-spectrum bandwidth

$R=$ data rate,

$E_{b}=$ bit energy,

$N_{0}=$ thermal (or background) noise density,

$I_{0}=$ maximum total acceptable interference density (interference power normalized by $W$ ), and

$f=$ ratio of other cell interference (at base station for given sector)-to-own sector interference ${ }^{2}$ then the condition for nonblocking is

$$
N_{u} E_{b} R(1+f)+N_{0} W \leq I_{0} W
$$

whence it follows that

$$
N_{u} \leq \frac{W / R}{E_{b} / I_{0}} \cdot \frac{1-\eta}{1+f}
$$

${ }^{2}$ In [7], a tight upper bound on other-cell interference is derived, which gives $f=0.55$ for the propagation parameters of interest. where

$$
\eta=N_{0} / I_{0}=0.1 \text { (nominally) } .
$$

In fact, however, none of the three assumptions above holds since

a) the number of active calls is a Poisson random variable with mean $\lambda / \mu$ (there is no hard limit on servers);

b) each user is gated on with probability $\rho$ and off with $1-\rho$

c) each user's required energy-to-interference $E_{b} / I_{0}$ ratio is varied according to propagation conditions to achieve the desired frame error rate ( $\approx 1$ percent).

For simplicity, we continue to assume that all cells are equally loaded (with the same number of users per cell and sector which are uniformly distributed over each sector).

With assumptions a), b), and c) replacing 1), 2), and 3), the condition for nonblocking, replacing (1) becomes

$$
\sum_{i=1}^{k} \nu_{i} E_{b i} R+\sum_{j}^{\text {other cells }} \sum_{i=1}^{k} \nu_{i(j)} E_{b_{i(j)}} R+N_{0} W \leq I_{0} W
$$

with $k$, the number of users/sector, being a Poisson random variable with mean $\lambda / \mu$; and $\nu$ being the binary random variable taking values 0 and 1 , which represents voice activity, with

$$
P(\nu=1)=\rho .
$$

Dividing by $I_{0} R$ and defining

$$
\epsilon=E_{b} / I_{0}
$$

the nonblocking condition (4) becomes

$$
Z \triangleq \sum_{i=1}^{k} \nu_{i} \epsilon_{i}+\sum_{j}^{\text {other cells }} \sum_{i=1}^{k} \nu_{i}^{(j)} \epsilon_{i}^{(j)} \leq(W / R)(1-\eta)
$$

where $\eta$ is given by (3). Hence, the blocking probability for CDMA becomes

$$
P_{\text {blocking }}=\operatorname{Pr}[Z>(W / R)(1-\eta)] .
$$

Setting this equal to a given value (nominally 1 percent) establishes the Erlang capacity of a CDMA cellular system. Again, we note that this is a "soft blocking" phenomenon which can be occasionally relaxed by allowing $I_{0} / N_{0}$, and consequently $1-\eta$, to increase.

Naturally, when condition (8) is exceeded, call quality will suffer. Thus, this probability is kept sufficiently low so that we can ensure high availability of good quality service. Conventional multiple access systems also are limited to providing good quality service only on the order of 90-99 percent of the time because of the variability of interference from just one or a few other users, in contrast with the CDMA case where quality depends on an average over the entire user population.

To evaluate this blocking probability, we must determine the distribution function of the random variable $Z$ which in 


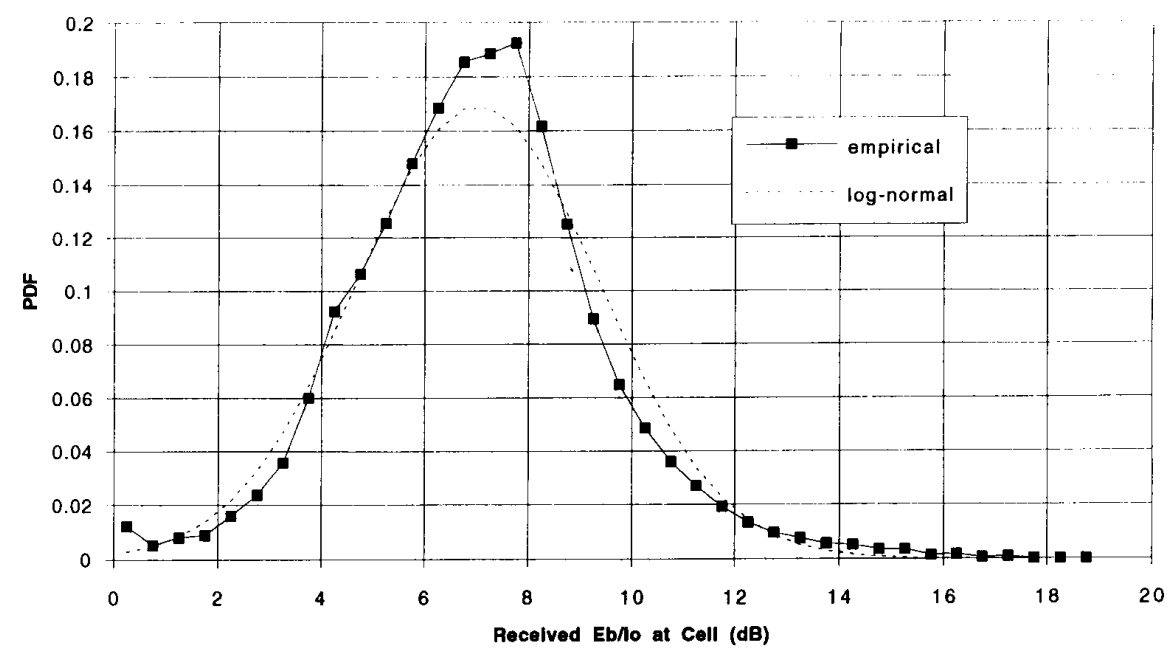

Fig. 1. Empirical $E_{b} / I_{0}$ probability density and log-normal approximation $(m=7.0 \mathrm{DB}$; sigma $=2.4 \mathrm{~dB})$.

turn depends on the random variables $\nu, k$, and $\epsilon$, representing voice activity, number of users in a sector, and $E_{b} / I_{0}$ of any user, respectively. The distribution of $\nu$ is given by (5). Since $k$ is Poisson, its distribution is given by

$$
p_{k}=\operatorname{Pr}(k \text { active users / sector })=\frac{(\lambda / \mu)^{k}}{k !} e^{-\lambda / \mu}
$$

where $\lambda$ and $\mu$ are the arrival and service rates as defined in the previous section.

On the other hand, $\epsilon$, the $E_{b} / I_{0}$ ratio of a single user, depends on the power control mechanism which attempts to equalize the performance of all users. It has been demonstrated that inaccuracy in power control loops are approximately lognormally distributed with a standard deviation between 1 and $2 \mathrm{~dB}$ [4]. However, since under some propagation conditions (e.g., with excessive multipath) higher than normal $E_{b} / I_{0}$ will be required to achieve the desired low error rates, the overall distribution, also log-normally distributed, will have a larger standard derivation. An example drawn from field trials with all cells fully loaded, in which $E_{b} / I_{0}$ is varied in order to maintain frame error rate below 1 percent is shown by the histogram of Fig. 1. As demonstrated by the dotted curve, the histogram is closely approximated by a log-normal probability density, with the mean and standard deviation of the normal exponent equal to 7 and $2.4 \mathrm{~dB}$, respectively. Hence, we shall use the log-normal approximation ${ }^{3}$

$$
\epsilon=10^{\times / 10}
$$

where $x$ is a Gaussian variable with mean $m \approx 7 \mathrm{~dB}$ and standard deviation $\sigma \approx 2.5 \mathrm{~dB}$. Note then that the first and

\footnotetext{
${ }^{3}$ In the following, we could use the exact empirical distribution in numerically computing the Chernoff bound (13). Using the log-normal approximation gives us more flexibility in obtaining a general analytical result. Actually, the approximation is slightly pessimistic (an upper bound) since it appears from Fig. 1 that the empirical histogram is slightly skewed to the left of the lognormal approximation with the same mean and standard deviation. Fig. 1 is typical of data from a large number of field tests conducted in widely varying terrain in a large number of cities and in several countries.
}

second moments of $\epsilon$ are given by

$$
\begin{gathered}
E(\epsilon)=E\left(e^{\beta x}\right)=\exp \left[(\beta \sigma)^{2} / 2\right] \exp (\beta m) \\
E\left(\epsilon^{2}\right)=E\left(e^{2 \beta x}\right)=\exp \left[2(\beta \sigma)^{2}\right] \exp (2 \beta m), \\
\beta=(\ln 10) / 10 .
\end{gathered}
$$

Although all moments exist, the moment generating function of $\epsilon$ does not converge; hence the ordinary Chernoff bound for the blocking probability (8) cannot be obtained. In Appendix I, we derive a modified Chernoff upper bound obtained by treating the upper end of the distribution of $\epsilon$ separately. The result for a single sector (no interference from other cells) is

$$
\begin{aligned}
P_{\text {blocking }}< & \operatorname{Min}_{\substack{s>0 \\
\tau>0}} \exp \left\{\rho(\lambda / \mu)\left[E\left(e_{T}^{s \epsilon^{\prime}}\right)-1\right]-s A\right\} \\
& +\rho(\lambda / \mu) Q(\tau / \sigma)
\end{aligned}
$$

where ${ }^{4}$

$$
\begin{gathered}
A \triangleq \frac{(W / R)(1-\eta)}{\exp (\beta m)}=\frac{(W / R)(1-\eta)}{E_{b} / I_{0_{\text {median }}}} \\
E\left(e^{s \epsilon_{T}^{\prime}}\right)=\int_{-\infty}^{\tau / \sigma} \exp \left[s e^{\beta \sigma \zeta}\right] e^{-\zeta^{2} / 2} d \zeta / \sqrt{2 \pi} \\
Q(\tau / \sigma)=\int_{\tau / \sigma}^{\infty} e^{-\zeta^{2} / 2} d \zeta / \sqrt{2 \pi} .
\end{gathered}
$$

This bound, obtained through numerical integration of (15), is plotted, for the single sector case, as the upper curve of Fig. 2.

${ }^{4}$ Note that $m$ is the mean of the exponent of the log-normal variable. Since $e^{\beta m}=10^{m / 10}$, this is the value of $E_{b} / I_{0}$ corresponding to the mean (dB) value, but it is not the true mean. It can be shown by symmetry that it is the median, while the true mean is related to it by (11). 


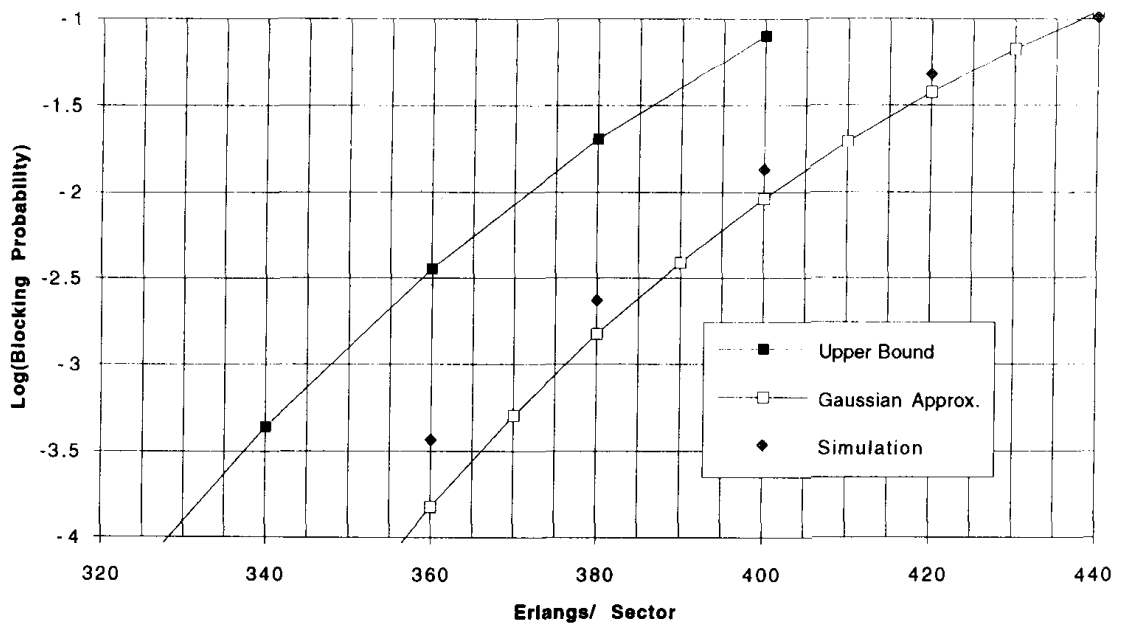

Fig. 2. Blocking probabilities for single cell interference (CDMA parameters: $W / R=1280$; voice act. $=0.4 ; I_{0} / N_{0}=10 \mathrm{~dB} ;$ median $E_{b} / I_{0}=7 \mathrm{~dB}$; sigma $=2.5 \mathrm{~dB})$.

A much simpler approach is to assume a central limit theorem approximation for $Z$ and to compute its mean and variance. Then

$$
P_{\text {blocking }} \approx Q\left[\frac{A-E\left(Z^{\prime}\right)}{\sqrt{\operatorname{Var} Z^{\prime}}}\right]
$$

where $Z^{\prime}=Z / \exp (\beta m)=Z /\left(E_{b} / I_{0}\right)_{\text {median }}$.

Now since $Z$ is the sum of $k$ random variables, where $k$ is itself a random variable, we have from [5], letting $\epsilon^{\prime}=\epsilon / \exp (\beta m)$

$$
\begin{gathered}
E\left(Z^{\prime}\right)=E(k) E\left(\nu \epsilon^{\prime}\right) \\
=(\lambda / \mu) \rho \exp \left[(\beta \sigma)^{2} / 2\right] \\
\operatorname{Var}\left(Z^{\prime}\right)=E(k) \operatorname{Var}\left(\nu \epsilon^{\prime}\right)+\operatorname{Var}(k)\left[E\left(\nu \epsilon^{\prime}\right)\right]^{2} .
\end{gathered}
$$

But since $k$ is a Poisson variable, $E(k)=\operatorname{Var}(k)=\lambda / \mu$, so that

$$
\begin{aligned}
\operatorname{Var}\left(Z^{\prime}\right) & =\lambda / \mu\left[E\left(\nu \epsilon^{\prime}\right)^{2}\right]=\lambda / \mu E\left(\nu^{2}\right) E\left(\epsilon^{\prime 2}\right) \\
& =(\lambda / \mu) \rho \exp \left[2(\beta \sigma)^{2}\right] .
\end{aligned}
$$

Using (18) and (20) in (17) yields the lower curve of Fig. 2.

It is commonly accepted that a Chernoff upper bound overestimates the probability by almost an order of magnitude. Moreover, a simulation involving on the order of one million frames was run to evaluate the tightness of the bound and the accuracy of the approximation. Results shown in Fig. 2 indicate excellent agreement with the approximation, with at most 1 percent discrepancy in Erlang capacity for the nominal 1 percent blocking probability. Thus, we shall henceforth use only central limit approximations, given the much greater ease of computation, which will allow us to obtain more general and more easily employed results. We note that for any specific case, a strict upper bound can always be obtained by numerically computing the Chernoff bound based on the empirical distribution of $E_{b} / I_{0}$ as given in (A.3)
TABLE I

Factor $f$ for Several Values of Sigma (Fourth Power Law)

\begin{tabular}{cc}
$\delta$ & $f$ \\
$(\mathrm{~dB})$ & \\
\hline 0 & 0.44 \\
2 & 0.43 \\
4 & 0.45 \\
6 & 0.49 \\
8 & 0.55 \\
10 & 0.66 \\
12 & 0.91 \\
\hline
\end{tabular}

of Appendix I. We note, however, that the approximation underestimates this upper bound by at most a few percent.

\section{General Erlang Capacity Formula INCLUDING OTHER CELL INTERFERENCE}

Before deriving the general formula, we consider the effect of users in other cells which are power controlled by other base stations. It has been shown both analytically [3] and by simulation that the interference from surrounding cells increases the average level at the base station under consideration by a fraction between $1 / 2$ and $2 / 3$ of that of the desired cell's users when the propagation attenuation is proportional to the fourth power of the distance times a log-normally distributed component whose differential standard deviation is $8 \mathrm{~dB}$. The results of an improved upper bound on the mean outer-cell interference fraction $f$ as derived in [7] and shown in Table I.

We note also that each user which is controlled by an othercell base station will also have an $E_{b} / I_{0}$ which is distributed according to the histogram of Fig. 1. Hence, this other-user interference can also be modeled by the same log-normal distribution as assumed for users of the desired cell. We can now modify (18) to accommodate other-cell users as well. The total number of other-cell users is generally much larger, but their average power is equivalent to that of $k f$ users. We shall therefore model them as such, recognizing that this is 
somewhat pessimistic since, with a larger number of smaller received power users, the mean power will remain the same but the variance will be reduced. In any case, accepting this approach as an overbound, we find that the mean and variance (18) and (20) are simply increased by the factor $1+f$. Thus, we may restate (17)-(20), when other-cell (power controlled) interfering users are included, as follows:

$$
\begin{gathered}
P_{\text {blocking }} \approx Q\left[\frac{A-E\left(Z^{\prime}\right)}{\sqrt{\operatorname{Var} Z^{\prime}}}\right] \\
E\left(Z^{\prime}\right)=(\lambda / \mu) \rho(1+f) \exp \left[(\beta \sigma)^{2} / 2\right] \\
\operatorname{Var}\left(\mathrm{Z}^{\prime}\right)=(\lambda / \mu) \rho(1+\mathrm{f}) \exp \left[2(\beta \sigma)^{2}\right] .
\end{gathered}
$$

Inverting (21) yields the quadratic equation

$$
x \alpha^{4}\left[Q^{-1}\left(P_{\text {blocking }}\right)\right]^{2}=[A-x \alpha]^{2}
$$

where $x=(\lambda / \mu) \rho(1+f)$, and $\alpha=\exp \left[(\beta \sigma)^{2} / 2\right]$, while $A$ is given by (14). Its solution is

$$
x=\frac{A}{\alpha}\left[1+\frac{\alpha^{3} B}{2}\left(1-\sqrt{1+\frac{4}{\alpha^{3} B}}\right)\right]
$$

where

$$
B=\frac{\left[Q^{-1}\left(P_{\text {blocking }}\right)\right]}{A}=\frac{\left(E_{b} / I_{0}\right)_{\text {median }}\left[Q^{-1}\left(P_{\text {blocking }}\right)\right]^{2}}{(W / R)(1-\eta)} .
$$

Using (24) and (25), this can be expressed as a formula for Erlang capacity

$$
\frac{\lambda}{\mu}=\frac{(1-\eta)(W / R) F(B, \sigma)}{\rho(1+f)\left(E_{b} / I_{0}\right)_{\text {median }}} \quad \text { Erlangs/Sector }
$$

where

$$
\begin{aligned}
F(B, \sigma)= & \exp \left[-(\beta \sigma)^{2} / 2\right] \\
& \cdot\left\{1+(B / 2) \exp \left[3(\beta \sigma)^{2} / 2\right]\right. \\
& \left.\cdot\left(1-\sqrt{1+4 \exp \left[-3(\beta \sigma)^{2} / 2\right] / B}\right)\right\}
\end{aligned}
$$

with $B$ given by (26). Fig. 3 is a plot of $\left(Q^{-1}\right)^{2}$-the factor of $B$ which depends on $P_{\text {blocking. Fig. } 4 \text { shows plots of }}$ $F(B, \sigma)$ as a function of $B$ for several values of $\sigma$, the standard deviation in decibels of the power-controlled $E_{b} / I_{0}$. We note finally that the Erlang capacity, as given by (27), is the same as the capacity predicted under ideal assumptions (2) but increased by the inverse of the voice activity factor $1 / \rho$ and decreased by the reduction factor $F(B, \sigma)$ given in Fig. 4. We note from Fig. 4 that a power control inaccuracy of $\sigma=2.5 \mathrm{~dB}$ causes only about as much capacity reduction as does the variability in arrival times and voice activity. Numerically, for $E_{b} / I_{0}=7 \mathrm{~dB}, \eta=0.1, W / R=31 \mathrm{~dB}$ and $P_{\text {blocking }}=1$ percent we obtain $B=0.024$. Then, from Fig. 4 , we note that $F(B, \sigma=2.5)=0.695$ while $F(B, \sigma=0)=$ 0.86 . Thus, it follows that the incremental loss due to power control inaccuracy $F(B, \sigma=2.5) / F(B, \sigma=0)=0.8$. This means that in this case a standard deviation of $2.5 \mathrm{~dB}$ in power control causes a reduction of only $1 \mathrm{~dB}$ in capacity. Fig. 3 and 4 also show that results are not very sensitive to the level of blocking probability. Doubling the latter only increases capacity by about 2 percent. Note finally that substituting $F(B, \sigma=2.5)=0.695$ in the general formula (27), we obtain, for the parameters noted above along with voice activity $\rho=0.4$ and other-cell factor $f=0.55$ (as obtained from Table I), an Erlang capacity $\lambda / \mu=258$ Erlangs/sector. Thus, comparison to Section II gives the ratio

$$
\frac{C_{\mathrm{CDMA}}}{C_{\mathrm{AMPS}}} \approx \frac{258}{12.34} \approx 20.9 \text {. }
$$

Based on the simulation results shown in Fig. 2, which suggest reduction of the approximate results by one to two percent, an estimate in excess of a twentyfold increase in Erlang capacity is thus justified.

\section{Designing for Minimum Transmitted Power}

In the preceding, the total received interference-to-noise $I_{0} / N_{0}$ was assumed fixed, and blocking probability was evaluated as a function of average user loading $\lambda / \mu$. Now suppose the blocking probability is fixed at 1 percent, but for each user loading $\lambda / \mu$, the minimum value of $I_{0} / N_{0}$ is determined to achieve this. This can be established by varying $\eta=N_{0} / I_{0}<$ 1 and determining from (27) the $\lambda / \mu$ value for which blocking probability equals 1 percent for each $I_{0} / N_{0}$ level.

More importantly, from $I_{0} / N_{0}$, we may determine the minimum value of received signal power-to-background noise for each user, and consequently the minimum transmitted power per user, given the link attenuation, the receiver sensitivity, and antenna gains. The minimum required received signal-tobackground noise per user $S /\left(N_{0} W\right)$ is obtained by equating the average sum of the per-user ratios weighted by the average voice activity factor to the total other user interference-to-noise ratio

$$
\rho \frac{\lambda}{\mu} \frac{S}{N_{0} W}=\left(\frac{I_{0}-N_{0}}{N_{0}}\right)
$$

Thus,

$$
\frac{S}{N_{0} W}=\frac{1}{\rho(\lambda / \mu)}\left(\frac{I_{0}}{N_{0}}-1\right)=\frac{(1 / \eta)-1}{\rho(\lambda / \mu)}
$$

where $1 / \eta=I_{0} / N_{0}$ is fixed, as noted above, and $\lambda / \mu$ is the resulting loading to achieve $P_{\text {blocking }}=0.01$ for this value of $\eta$, as given by (26)-(28). Fig. 5 shows the per-user signalto-background noise ratio as a function of the relative Erlang capacity for the same parameters used in the last section. The inverse of $\eta$, the interference-to-noise density $I_{0} / N_{0}$ ranges from 1 to $10 \mathrm{~dB}$ as the capacity varies over a factor of 8 . The important point is that the per-user power can be reduced by about $8 \mathrm{~dB}$ for lightly loaded cells. It also helps to justify the choice of $I_{0} / N_{0}=10 \mathrm{~dB}$ for blocking when all cells are heavily loaded, since per-user power requirements increase rapidly above this point. 


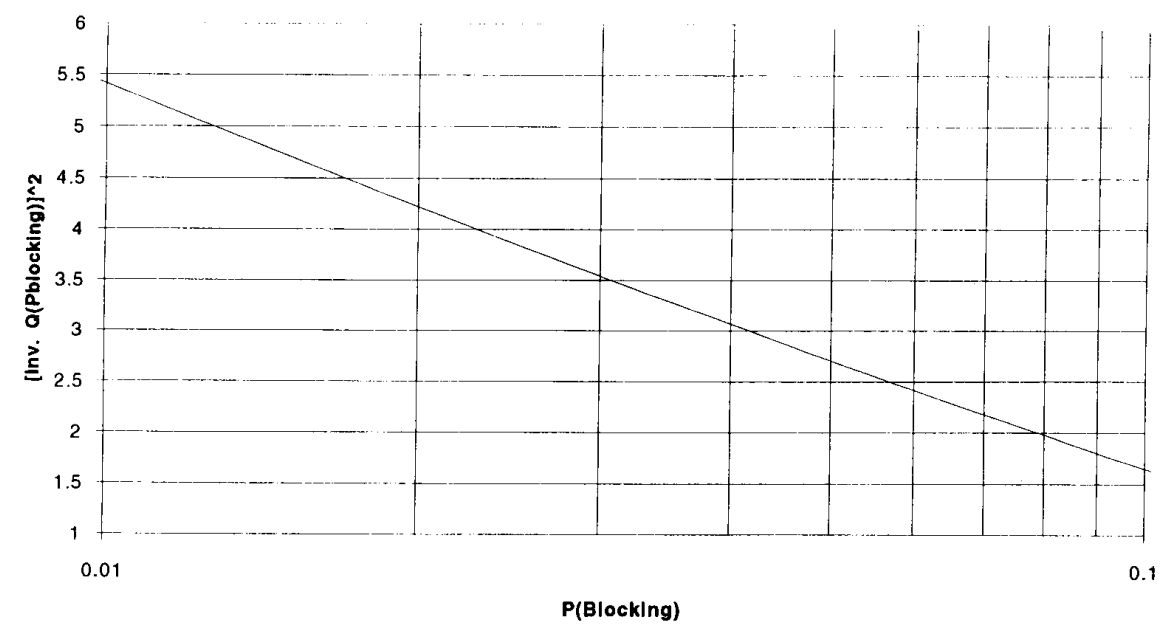

Fig. 3. Variable factor in $B$ as a function of $P$ (blocking).

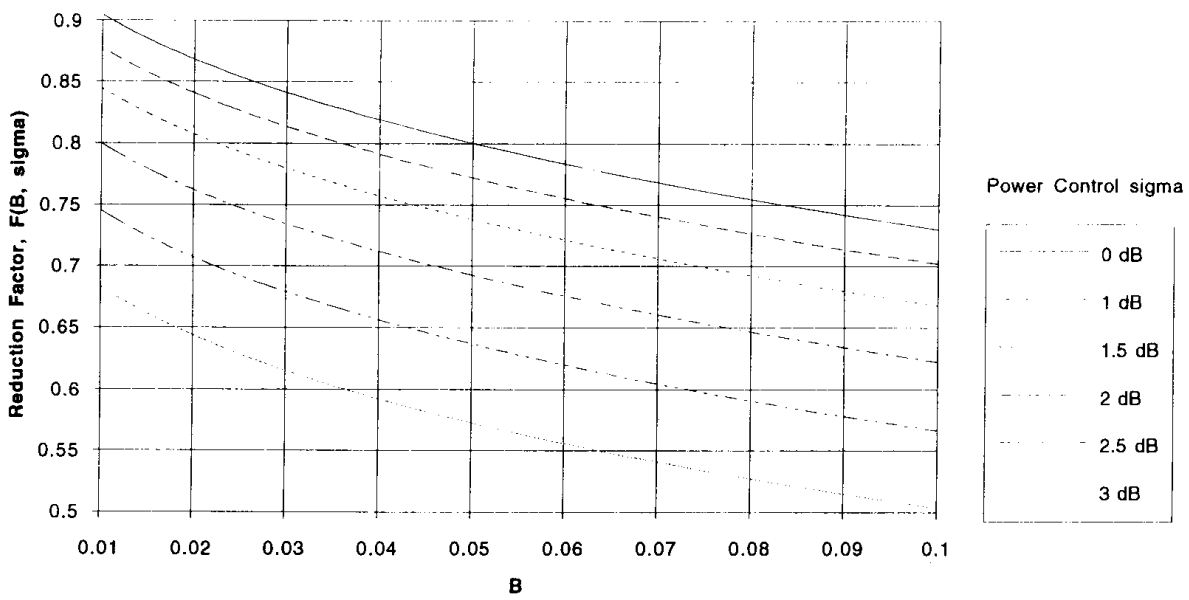

Fig. 4. Erlang capacity reduction factors.

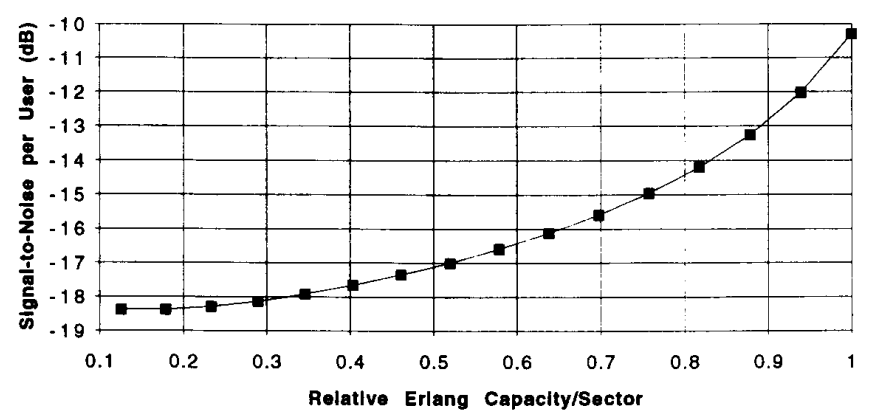

Fig. 5. Signal-to-noise per user as a function of relative sector capacity.

\section{INITIAL ACCESS}

Prior to initiating a call on the reverse link, a user must signal a request to the base station. In conventional systems, a time or frequency slot is allocated for the purpose of access request, and a protocol is provided for recovering from collisions which occur when two or more new users send access requests simultaneously. In CDMA, in which the allocated commodity is energy rather than time or frequency, access requests can share the common channel with ongoing users. The arrival rate of user requests is taken to be $\lambda$ calls/s, which is the same as that for ongoing calls under the assumption that all requests are eventually served. Since newly 
arriving users are not power controlled until their requests are recognized, the initial power level will be taken to be a random variable uniformly distributed from 0 to a maximum value, corresponding to a (bit) energy level of $E_{M}$. Thus, the initial access energy level is $\gamma E_{M}$ where $\gamma$ is a random variable with probability density function

$$
p(\gamma)= \begin{cases}1, & 0<\gamma<1 \\ 0, & \text { otherwise }\end{cases}
$$

If this initial power level is not sufficient for detection, ${ }^{5}$ and hence acknowledgment is not received, the user increases his power in constant decibel steps every frame until his request is acknowledged. Thus, the initial access user's power grows exponentially (i.e., linearly in decibels) with time, taken to be continuous since the frame time is only tens of milliseconds long. Hence, the energy as a function of time for initial access requests is

$$
E(t)=\gamma E_{M} e^{\delta t}
$$

where $\delta$ is fixed. Letting $\tau$ denote the time required for an initial access user to be detected, it follows from (30) to (31) that

$$
\begin{aligned}
\operatorname{Pr}(\tau>T) & =\operatorname{Pr}\left(\gamma E_{M} e^{\delta T}<E_{M}\right) \\
& =\operatorname{Pr}\left(\gamma<e^{-\delta T}\right)=e^{-\delta T}, \quad T>0
\end{aligned}
$$

where the last equation follows from the fact that $\gamma$ is uniformly distributed on the unit interval.

Thus, the "service time" for any initial access requests (time required for acceptance) for all users is exponentially distributed with mean $1 / \delta \mathrm{s} /$ message. The consequences of this observation are very significant. We have thus shown that with Poisson distributed arrivals and exponentially distributed service time, the output distribution is also Poisson. This then guarantees that the distribution for users initiating service is the same as for newly accessing users. Furthermore, at any given time, the power distribution for users which have not yet been accepted is also uniform. As for the service time distribution, according to (32), this is exponential with mean service time equal to $1 / \delta$.

Thus, the total interference of (4) is augmented by the interference from initial accesses. When normalized as in (18), this introduces an additional term in the mean and variance of $Z^{\prime},(18)$ and (20), respectively. Calling this $Z^{\prime \prime}$, we have, ignoring for the moment surrounding cells,

$$
E\left(Z^{\prime \prime}\right)=E\left(Z^{\prime}\right)+(\lambda / \delta) E\left(\gamma E_{M} / I_{0}\right) / e^{\beta m}
$$

$$
\operatorname{Var}\left(Z^{\prime \prime}\right)=\operatorname{Var}\left(Z^{\prime}\right)+(\lambda / \delta) E\left(\gamma^{2} E_{M} / I_{0}\right) / e^{2 \beta m}
$$

where $\gamma$ is uniformly distributed on the unit interval and is independent of $E_{M} / I_{0}$. Thus $E(\gamma)=1 / 2$ and $E\left(\gamma^{2}\right)=$

\footnotetext{
${ }^{5}$ We assume initially that the access request is detected (immediately) when the initial access user's energy reaches $E_{M}$, but it remains undetected until that point. Below, we shall take $E_{M}$ to be itself a random variable, with distribution similar to $E_{b}$. It should also be noted that initial access detection is performed on an unmodulated signal, other than for the user's pseudorandom code which is known to the base station. It should thus be more robust than the demodulator performance at the same power level.
}

1/3. We take $E_{M} / I_{0}=\theta\left(E_{b} / I_{0}\right)$, and hence log-normally distributed with the same $\sigma$ as $E_{b} / I_{0}$. Then $E\left(E_{M} / I_{0}\right)=$ $\theta E(\epsilon), E\left[\left(E_{M} / I_{0}\right)^{2}\right]=\theta^{2} E\left(\epsilon^{2}\right)$.

If surrounding cells support the same coverage load as the given cell, initial accesses will also produce the same relative interference. Introducing this term as well, we obtain, from (33) and (34),

$$
\begin{aligned}
E\left(Z^{\prime \prime}\right)= & \left\{(\lambda / \mu) \rho \exp \left[(\beta \sigma)^{2} / 2\right]\right. \\
& \left.+(\lambda / \delta)(1 / 2) \theta \exp \left[(\beta \sigma)^{2} / 2\right]\right\}[1+f] \\
= & \rho(\lambda / \mu)\left(1+\frac{\theta \mu}{2 \rho \delta}\right) \exp \left[(\beta \sigma)^{2} / 2\right][1+f] \\
\operatorname{Var}\left(Z^{\prime \prime}\right)= & \left\{(\lambda / \mu) \rho \exp \left[2(\beta \sigma)^{2}\right]\right. \\
& \left.+(\lambda / \delta)(1 / 3) \theta^{2} \exp \left[2(\beta \sigma)^{2}\right]\right\}[1+f] \\
= & \rho(\lambda / \mu)\left(1+\frac{\theta^{2} \mu}{3 \rho \delta}\right) \exp \left[2(\beta \sigma)^{2}\right][1+f] .
\end{aligned}
$$

If we take the arbitrary scale factor $\theta=3 / 2$ - a reasonable choice since this places the detection bit energy level at 50 percent higher than the operating bit energy level-we find that the effect is to increase the Erlang level $(\lambda / \mu)$ by the factor

$$
F=1+\left(\frac{3}{4 \rho}\right)\left(\frac{\mu}{\delta}\right)=1+1.88(\mu / \delta) \text { for } \rho=0.4 .
$$

Note that $\mu / \delta$ is the ratio of mean detection time-to-mean message duration, which should be very small. If, for example, $\mu / \delta=0.0055$, corresponding to a mean access time of $1 \mathrm{~s}$ (50 frame) ${ }^{6}$ for a $3 \cdot \mathrm{min}$ mean call duration, this means that the effect of initial accesses is to reduce the Erlang traffic by about 1 percent (a negligible cost considering the notable advantages).

\section{CONCLUSIONS}

The foregoing analysis represents a conservative estimate of the Erlang capacity of direct sequence spread-spectrum CDMA. Classical blocking was replaced by the condition that the total interference exceeds the background noise by $10 \mathrm{~dB}$, and Erlang capacity was defined as the traffic load corresponding to a 1 percent probability that this event occurs. The three random variables which contribute to this event were modeled as follows:

1) Poisson traffic arrival, exponentially distributed message length, and arbitrarily many servers $(M / M / \infty$ in the terminology of queuing theory [1]);

2) voice activity factor $\rho$ equal to 40 percent, as established by extensive experimental measurements on ordinary speech conversations [6];

\footnotetext{
${ }^{6}$ Note that at a data rate of $9600 \mathrm{bits} / \mathrm{s}$, each $20 \mathrm{~ms}$ voice frame contains 192 bits. For $E_{b} / I_{0} \approx 7 \mathrm{~dB}$, the frame energy-to-interference level is about $32 \mathrm{~dB}-$ an ample value to virtually assure detection in one frame.
} 
3) individual user's received bit energy-to-interference density $E_{b} / I_{0}$ whose distribution was determined empirically from field measurements and supported by analytical results [4].

The main conclusion is that the Erlang capacity of CDMA is about 20 times that of AMPS. This is based on establishing the blocking condition as the event that the total interferenceto-noise-ratio exceeds $10 \mathrm{~dB}$. This "soft" blocking condition can be relaxed to allow a maximum ratio of $13 \mathrm{~dB}$. for example, for particularly heavily loaded sectors. When traffic is light, much lower interference-to-noise ratios can be imposed which translate into much lower mobile transmitted powers, a particularly valuable feature in prolonging battery life for portable subscriber units.

Finally, a significant byproduct of spectrum sharing is the very small Erlang capacity overhead required to accommodate initial access requests along with ongoing traffic, as demonstrated in the last section.

\section{APPENDIX}

\section{Modified CHERnoff BOUNd For A Single Sector}

Starting with (8), without considering outer cells, we may bound ( 8 ) by introducing an arbitrary threshold on each term $\nu_{i} \epsilon_{i}^{\prime}$, where $\epsilon^{\prime}=\epsilon / e^{\beta m}$. Thus,

$$
\begin{aligned}
P_{\text {blocking }}= & \operatorname{Pr}\left(\sum_{i=1}^{k} \nu_{i} \epsilon_{i}>(W / R)(1-\eta)\right) \\
= & \operatorname{Pr}\left(\sum_{i=1}^{k} \nu_{i} \epsilon_{i}^{\prime}>A\right) \\
< & \operatorname{Pr}\left(\sum_{i=1}^{k} \nu_{i} \epsilon_{i}^{\prime}>A \mid \nu_{i} \epsilon_{i}^{\prime}<T \text { for all } i\right) \\
& +\operatorname{Pr}\left(\nu_{i} \epsilon_{i}^{\prime}>T \text { for any } i\right)
\end{aligned}
$$

where $A$ is given by (14).

Bounding the first term by its (conditional) Chernoff bound and the second by a union bound, we have, using (9),

$$
\begin{aligned}
P_{\text {blocking }}< & E_{k} E_{\epsilon^{\prime}} E_{\nu}\left(\left[\exp \left(s \nu \epsilon^{\prime}\right)\right]^{k} \mid \epsilon^{\prime}<T\right) e^{-s A} \\
& +E_{k}\left[\sum_{i=1}^{k} \operatorname{Pr}\left(\epsilon_{i}^{\prime}>T\right) \operatorname{Pr}\left(\nu_{i}=1\right)\right] \\
= & E_{k}\left(\left[\rho E_{e}^{s \epsilon^{\prime}}+(1-\rho)\right]^{k} \mid \epsilon^{\prime}<T\right) e^{-s A} \\
& +\rho(\lambda / \mu) \operatorname{Pr}\left(\epsilon^{\prime}>T\right) \\
= & \exp \left[\rho(\lambda / \mu) E\left(e^{s \epsilon^{\prime}} t\right)-1\right] e^{-s A} \\
& +\rho(\lambda / \mu) \operatorname{Pr}\left(\epsilon^{\prime}>T\right) \\
E e^{s \epsilon_{T}^{\prime}}= & \int_{-\infty}^{(\operatorname{In} T) / \beta} \exp \left(s e^{\beta \xi}\right) \frac{e^{-\xi^{2} /\left(2 \sigma^{2}\right)}}{\sqrt{2 \pi} \sigma} d \xi \\
= & \int_{-\infty}^{\tau / \delta} \exp \left(s e^{\beta \sigma \zeta}\right) e^{-\zeta^{2} / 2} d \zeta / \sqrt{2 \pi}
\end{aligned}
$$

where $\tau=(\ln T) / \beta$, and

$$
\begin{aligned}
\operatorname{Pr}\left(\epsilon^{\prime}>T\right) & =\operatorname{Pr}(\xi>(\ln T) / \beta) \\
& =\int_{(\ln T) / \beta}^{\infty} e \frac{-\xi^{2} / 2 \sigma^{2}}{\sqrt{2 \pi} \sigma}=Q(\tau / \sigma)
\end{aligned}
$$

Inserting (A.4) and (A.3) into (A.2), and minimizing $s>0$ and $\tau>0$, yields (13) $-(16)$.

The total interference is then the sum of (B.3) and (B.7), where the latter is integrated over all regions not including the six central region pairs $A$ and $B$. The result equals $N_{u}(1+f)$. By performing this numerical integration, the othercell interference factor $f$ was obtained for various values of $\mu$ and $\sigma_{0}$, as shown in Table I.

\section{ACKNOWLEDGMENT}

The authors are grateful to E. Zehavi for suggesting the approach to the modified Chernoff bound of the Appendix, and to the highly motivated QUALCOMM team whose tireless efforts proved the feasibility of CDMA technology, and particularly of the power control methods which led to the empirical results shown in Fig. 1.

\section{REFERENCES}

[1] D. Bertsekas and R. Gallager, Data Networks. Englewood Cliffs, NJ: Prentice-Hall, 1987.

[2] G. R. Cooper and R. W. Nettleton, "A spread spectrum technique for high capacity mobile communications," IEEE Trans. Veh. Technol., vol. VT27, pp. 264-275, Nov. 1978 .

[3] K. S. Gilhousen, I. M. Jacobs, R. Padovani, A. J. Viterbi, L. A. Weaver, Jr., and C.E. Wheatley, III, "On the capacity of a cellular CDMA system," IEEE Trans. Veh. Technol., vol. 40, May 1991.

[4] A.J. Viterbi, A.M. Viterbi, and E. Zehavi, "Performance of powercontrolled wideband terrestrial digital communications," to appear in IEEE Trans. Commun., 1993.

[5] W. Feller, An Introduction to Probability Theory and Its Applications, Vol. I, 2nd ed. New York: Wiley, 1957.

[6] P. T. Brady, "A statistical analysis of on-off patterns in 16 conversations," Bell Syst. Tech. J., vol. 47, pp. 73-91, Jan. 1968.

[7] A. J. Viterbi, A. M. Viterbi, and E. Zehavi, "Other-cell interference in cellular power-controlled CDMA," IEEE Trans. Commun., to appear.

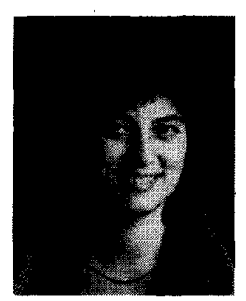

Audrey M. Viterbi received the B.S. degree in electrical engineering and computer science and the B.A. degree in mathematics from the University of California, San Diego, in 1979. She received the M.S. and Ph.D. degrees in electrical engineering from the University of California, Berkeley, in 1981 and 1985 , respectively.

She was an Assistant Professor of Electrical and Computer Engineering at the University of California, Irvine, from 1985 to 1990 . Since 1990 she has been a Staff Engineer at Qualcomm, Inc., San Diego, where she is currently involved in the development of the CDMA cellular telephone system. Her professional interests are in the area of modeling and performance evaluation of computer-communication networks and communications systems. 


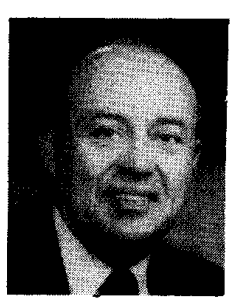

Andrew J. Viterbi received the S.B. and S.M degrees from the Massachusetts Institute of Technology, Cambridge, in 1957, and the Ph.D. degree from the University of Southern California in 1962

In his first employment after graduating from M.I.T., he was a member of the project team at C.I.T. Jet Propulsion Laboratory which designed and implemented the telemetry equipment on the first successful U.S. satellite, Explorer I. In the early 1960's at the same laboratory, he was one of the first communication engineers to recognize the potential and propose digital transmission techniques for space and satellite telecommunication systems. As a Professor in the UCLA School of Engineering and Applied Science from 1963 to 1973, he did fundamental work in digital communication theory and wrote two books on the subject, for which he received numerous professional society awards and international recognition. In 1968 he co-founded LINKABIT Corporation and served as its Executive Vice President from 1974 to 1982, and as President from 1982 to 1984 . On July 1,1985 , he co-founded and became Vice Chairman and Chief Technical Officer of QUALCOMM, Inc., a company specializing in mobile satellite and terrestrial communication and signal processing technology.
Since 1975 he has been associated with the University of California, San Diego, and since 1985, as Professor (quarter time) of Electrical and Computer Engineering.

Dr. Viterbi is a member of the U.S. National Academy of Engineering. He is past Chairman of the Visiting Committee for the Electrical Engineering Department of Technion, Israel Institute of Technology, past Distinguished Lecturer at the University of Illinois and the University of British Columbia, and he is presently a member of the M.I.T. Visiting Committee for Electrical Engineering and Computer Science. In 1986 he was recognized with the Annual Outstanding Engineering Graduate Award by the University of Southern California, and in 1990 he received an honorary Doctor of Engineering Degree from the University of Waterloo, Ont. Canada. He presented the Shannon Lecture at the 1991 International Symposium on Information Theory. He has received three paper awards, culminating in the 1968 IEEE Information Theory Group Outstanding Paper Award. He has also received four major international awards: the 1975 Christopher Columbus International Award (from the Italian National Research Council sponsored by the City of Genoa); the 1984 Alexander Graham Bell Medal (from IEEE, sponsored by AT\&T) "for exceptional contributions to the advancement of telecommunications;" the 1990 Marconi International Fellowship Award; and was co-recipient of the 1992 NEC C\&C Foundation Award. 